Aus der Universitäts-Poliklinik für Ohren-, Nasen- und Kehlkopfkranke in Göttingen.

Direktor: Prof. Dr. Lange.

\title{
Beitrag zur Entstehung der Zysten am Naseneingang.
}

\author{
Von Professor W. Uffenorde, Göttingen.
}

Mit 1 Abbildung.

Von Grünwald, Blumenthal und Klestadt sind Zysten, die sie in der Umgebung des Naseneinganges beobachteten, auf Entwicklungsstörungen bei der Verschmelzung der primären embryonalen Gesichtsfortsätze zurückgeführt worden. Sie sind von Klestadt ${ }^{1}$ ) als Gesichtsspaltzysten bezeichnet, und diese Auffassung und Bezeichnung ist von einer Reihe von Autoren inzwischen für ähnliche Fälle übernommen worden.

Neuerdings hat nun Brüggemann²) auf die Beobachtung eines ähnlichen Falles hin sich eingehend mit der Entstehung der Zysten am Naseneingang beschäftigt und darauf hingewiesen, daß sie keineswegs auf eine eindeutige Entstehungsursache zurückzuführen seien. Nach Erörterung der verschiedenen Ursachen und Ausgangspunkte glaubt er die Gesichtsspaltzysten, wenn nicht überhaupt für unwahrscheinlich, so doch jedenfalls für selten halten zu müssen, und beruft sich auf das Urteil von Peter, der nach der Beschreibung von den Zysten diese Herkunft bezweifele.

Da also nach Lage der Dinge nicht nur die einzelnen Fälle in genetischer Hinsicht in Zweifel gezogen werden, sondern sogar die Möglichkeit, ob die Gesichtsspaltstörung überhaupt eine ursächliche Rolle spielen kann, ganz allgemein noch nicht als gesichert erscheint, möchte ich auf Grund des Berichtes von einem, vor kurzem beobachteten einschlägigen Falle nochmals auf diese Verhältnisse zurückkommen.

1) Gesichtspaltzysten. Demonstration. Breslauer chirurg. Ges. 2i. VII r9I3. Berl. klin. Wochenschr. I913, 96.

2) Zyste als Folge von Entwicklungsstörungen im Naseneingang. Brüggemann, Arch. f. Laryng. 33, S. 193 . 
Die Mitteilung mag auch durch die Seltenheit, namentlich in der Form, wie sie unser Fall aufweist, gerechffertigt erscheinen. Brüggemann hat insgesamt nur 52 Fälle von Zystenbildung am Naseneingang verschiedensten Ursprunges - fast ausschließlich bei Frauen - aus der Literatur sammeln können.

Der Fall ist kurz folgender:

Die 30 jährige Krankenschwester B. klagte seit etwa 5 Jahren über Schmerzen, die linksseitig meist anfallsweise in der Oberkiefergegend und auch nach den Augen und der Stirn ausstrahlend auftraten. Sie waren in der letzten Zeit, namentlich nachts unerträglich gewesen. Wegen der dauernden Zahnschmerzen ist die Kranke oft vergeblich zahnärztlich behandelt worden, und es sind schon am Oberkiefer links vier Zähne gezogen worden, die nur geringe Veränderungen geboten hätten. Die Oberlippe sei allmählich kürzer geworden und der Nasenflügel links verbreitert. Ja, die ganze Nase schiene nach rechts hinüber verbogen. Als Kind ist sie von einem Hund in die Nase gebissen worden; davon rühre noch eine Narbe an der Nasenwurzel her. Außerdem leide sie oft an Schnupfen und verstopfter Nase beiderseits.

Der Befund bestätigt die Angaben der Kranken. Die bewegliche Nase erschien nach rechts etwas hinüber gedrängt und der Nasenflügelansatz links nach außen und oben verschoben. Die Oberlippe war links etwas vorgetrieben, man fühlte darunter eine prall-elastische Schwellung. Die Weichteile waren im übrigen reizlos und nicht besonders schmerzempfindlich. Hinter dem Naseneingang links sah man unten und seitlich die Vestibularwand buckelförmig vorgewölbt; die Schleimhaut war im übrigen unverändert und von einem Gefäß durchzogen. Die vorgewölbte Partie ließ sich leicht mit der Sonde eindrücken.

In der übrigen Nasenhöhle war neben einer hochgradigen Verbiegung der Nasenscheidewand nach rechts eine lappige Schwellung der unteren Muscheln nachzuweisen: Veränderungen, die für die Verlegung der Nase und für die Neigung zu Schnupfen verantwortlich zu machen waren. Sie bestanden als selbstständige Erkrankung, unabhängig und neben der eben beschriebenen, und wurden durch entsprechende Eingriffe beseitigt. Im Röntgenbilde hob sich deutlich links neben der unteren seitlichen Umrandung der Apertura piriformis ein rundlicher Schatten ab, der der Zyste entsprach.

Diese ließ sich vom Mundvorhof aus in örtlicher Betäubung glatt und lückenlos ausschälen, ohne daß irgendeine Knochenkapsel oder eine Beziehung zum Zahnşystem oder zum Naseninnern sich verriet. Die Zyste war etwa kleinhaselnußgroß und wies eine ausgesprochene Birnenform mit abgebogenen Halsteile auf. Ihr Körper lag auf der Wurzel des Processus alveolaris über den seitlichen Schneidezähnen links oben urd hatte einen deutlichen Druckschwund am Knochen in Form einer umschriebenen Mulde verursacht. Der Zystenhals umzog den seitlichen und unteren Aperturrand, wobei sich der Stiel nach oben bis zum Ansatze des Stirnfortsatzes vom Oberkiefer verfolgen ließ.

Die sofort geschlossene Wunde heilte per primam. Die Beschwerden schwanden in kurzer Zeit völlig.

Der Inhalt der Zyste war klar, stark schleimhaltig und enthielt keine wesentlichen zelligen Beimengungen und kein Cholestearin.

Die histologische Untersuchung der Zystenwand in Serienschnitten ergab: die dünne Zystenwand ist, von einem ziemlich weit verzweigten Venennetz umgeben. Irgendwelche entzündliche Veränderungen fehlen. Die Auskleidung besteht, bis in den Stiel hinein, vorwiegend aus einem zweireihigen, wechselnd 
hohen Zylinderepithel mit basalständigen Kernen, in dem ziemlich reichlich becherartige, schleimenthaltende Zellen eingestreut sind. Es sind keinerlei drüsige Bildungen festzustellen. Flimmerbesatz kann nirgends eindeutig nachgewiesen werden.

An einzelnen Stellen der Wandung nahe dem unteren Ende finden sich starke Verdickungen des Epithels mit großen vieleckigen Zellen von Plattenepithelcharakter, die $z$. T. von einer Lage niedriger Zylinderzellen uberschichtet sind.

Wenn wir nun an die Frage herantreten: Wie ist diese Zyste entstanden, so können wir wohl die Möglichkeit der Zahnzyste einmal mangels jeder Andeutung einer Knochenhülle und irgendwelcher Beziehung zu den Zähnen und andererseits bei dem histologischen Befunde an Wand und Epithel, von vornherein ausschließen. Ebenso kann der Hundebiß in die Nase, von dem nur eine kleine Narbe am Nasenrücken herrührt, nicht die äußere Veranlassung zu der Störung gewesen sein. In diesem Falle müßten wir auch bei Versenkung von Epidermis eine atheromatöse Bildung erwarten. Immerhin wäre nach Traumen und operativen Eingriffen, durch Verlagerung von Schleimhaut, z. B. der Nasen- und Nebenhöhlen auch das Entstehen einer Mukoidzyste durchaus möglich.

Angesichts der Größe und Lage der Zyste kann es sich hier auch nicht um eine Retentionszyste von einer Schleimdrüse aus oder um eine entzündlich entstandene Knorpelhautzyste (Erweichungszyste) (Zarniko) gehandelt haben.

Weiter wäre an einem Zusammenhang der Zyste mit der Entwicklung des Gesichtsschädels zu denken. In dieser Hinsicht wollen wir zunächst die Herkunft vom Ductus nasopalatinus (Grünwald) erwägen. Der Ductus nasopalatinus entsteht aus einem, im embryonalen Leben vorübergehend unterbrochenen (Peter $\left.{ }^{1}\right)$ ) Epithelstrang zwischen Nase und Mund an der Vereinigungsstelle von Oberkiefer und mittlerem Nasenfortsatz, dort, wo in der Mittellinie primärer und sekundärer Gaumen zusammenwachsen.

Dagegen spricht ebenfalls die Lage und Ausdehnung der ganz seitlich entwickelten Zyste. Die vom Ductus nasopalatinus ausgehenden derartigen Bildungen müßten mittwärts am Nasenboden gelegen sein. Sie könnten erst sekundär bei größerem Umfange sich in den Vorhof hineinentwickeln.

Von Grosser ist der Nachweis erbracht worden, daß die bei Tieren oft stark entwickelte Glandula nasalis lateralis (Stenson) auch beim Menschen in verkümmerter Weise angelegt werden kann. Der bei Tieren nicht selten weit nach vorn ziehende Ausführungsgang liegt dorsalwärts oberhalb des Nasoturbinale. Das ganze Gebilde geht aus

1) Peter, Atlas der Entwicklung der Nase und des Gaumens beim Menschen mit Einschluß der Entwicklungsstörungen. Fischer, Jena I91 $_{9}$. 
einer Ausstülpung der Nasenschleimhaut hervor. Grosser hat es bei I5 menschlichen Embryonen $9 \mathrm{mal}$ als einen kleinen rudimentären Gang gefunden, der später wieder seine Lichtung verliere und einen soliden Zellhaufen darstelle. Die daraus möglicherweise zur Entstehung gelangenden Zysten würden am Agger nasi (das beim Menschen verkümmerte Nasoturbinale) im oberen Nasenflügelteil sitzen, nie aber im unteren Nasengang oder am Vorhof. Auch diese Genese kann also hier nicht in Betracht kommen. Sie ist übrigens bislang noch nicht durch einen Fall belegt worden; sondern nur ihre Möglichkeit von Brüggemann theoretisch erörtert.

Es sind nun noch zwei andere entwicklungsgeschichtlich interessante Möglichkeiten der Herkunft in Betracht zu ziehen: einmal die Entstehung der Zysten vom Ductus nasolacrimalis aus und zweitens ihre Entwicklung aus Zellverbänden, die nach der Vereinigung der primären Gesichtsfortsätze im Bindegewebe liegen geblieben sind. Die erstere glaubt namentlich Brüggemann bewerten zu müssen. Er hält sie für die häufigste Form und verweist auf die Dissertation von Tüffers ${ }^{1}$ ) über die Entwicklung des nasalen Endes des Tränennasenkanals. Bei den unter Peter s Leitung angestellten Untersuchungen ist nachgewiesen worden, da $B$ sich das bei den einzelnen Säugetieren ganz verschieden verhält. Bald findet sich nur eine Öffnung ganz vorne am Nasenvorhof, bald sind zwei Öffnungen vorhanden, eine vorn am Nasenvorhof und die andere hinter der dann anzunehmenden Anlegestelle des Duktus an dem unteren Nasengange. Schließlich kann auch nur die hintere Öffnung vorhanden sein, und das vordere Duktusstück mit der vorderen, stammesgeschichtlichen älteren Öffnung ist verkümmert. Die Gründe für dieses merkwürdige Verhalten vermag Tüffers nicht sicher anzugeben. Vielleicht ist der Verlust des vorderen Endes auf die Entwicklung der Nase nach oben zurückzuführen. Jedenfalls ist beim Menschen nur die hintere Öffnung ausgebildet, sie liegt nahe dem Dach des unteren Nasenganges, also unter der unteren Muschel. Nun ist schon von Arlt, besonders aber weiterhin von Bochdalek, Monesi u. a. eine von der. Tränennasengangsöffnung nach unten und vorn verlaufende, etwas mittewärts umbiegende Rinne oder Furche in der Schleimhaut gesehen, die etwa bis zur mittleren Höhe des unteren Nasenganges herunterzog (Merkel). In einzelnen Fällen ist auch noch eine zweite Öffnung geunden worden, wo dann die Strecke zwischen den beiden Öffnungen am Tränennasenkanal durch eine Schleimhautbrücke überdacht war. Wenn Tüffers nun aber nach den Befunden von Bochdalek und Monesi seine Untersuchungsergebnisse auf den Menschen übertragen zu können glaubt, so sind wohl Zweifel berechtigt, ob es

1) Tüffers, Die Entwicklung des nasalen Endes des Tränennasenganges bei einigen Säugetieren. Diss., Greifswald I9r3. 
sich bei den in Anspruch genommenen Bildungen um ein rückschläglich entwickeltes vorderes Ende des Tränennasenkanals handelt. Dieses müßte ja nach Einmündung in den unteren Nasengang nach außen und vorne ziehen. Der nasale Teil des Kanals schlingt sich z. B. beim Kaninchen um die Lamina transversalis anterior nach außen herum, nachdem er sich dem Schleimhautsack der Nase sehr genähert hat, - dort wo die hintere alleinige Öffnung des Tränennasenkanals beim Menschen entsteht. Wo seine vordere Öffnung beim Menschen $\mathrm{zu}$ finden wäre; - wenn eine solche überhąupt in Betracht kommen
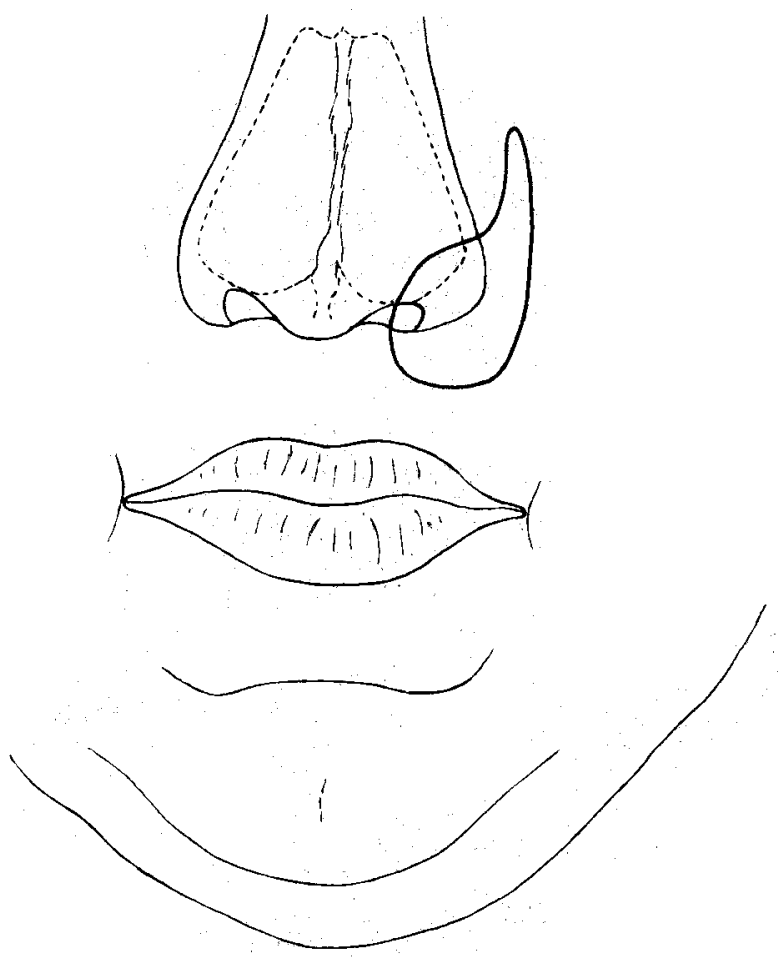

Abb. I. Ausdehnung und Lage der Zyste seitlich von der Nase, links,

sollte, - ist allerdings schwer zu sagen, da die Lamina transversalis anterior bei ihm fehlt. Bislang ist jedenfalls wohl beim Menschen das stammesgeschichtlich interessante vordere Stück des Tränennasenkanals noch niemals nachgewiesen worden. Auch die Bejahung der von Peter S. 68 in seinem Atlas aufgeworfenen Frage: „ob Kanäle, die beim Menschen in seltenen Fällen von der Öffnung des Tränennasenkanals bis in den Canalis incisivus verfolgt werden konnten und dort in einigen Fällen durchbrachen, diesem vorderen, den meisten Säugern zukommenden, Kanalstück entsprechen", ist nach Prof. 
M. Voit, der mir in freundlichster Weise die Verhältnisse an seinen Serien und Modellen durchging, als unwahrscheinlich zu bezeichnen. Zudem würde dann auch dieses Kanalstück noch innerhalb der Apertura piriformis $\mathrm{zu}$ finden sein.

Wenn also auch theoretisch die Möglichkeit, daß sich beim Menschen aus einem vorderen, verkümmert angelegten Ende des Tränennasenkanals eine Zyste entwickeln kann, zuzugeben ist, so ist die Annahme doch zurzeit noch als ganz hypothetisch zu betrachten. Mangels irgendwelcher gesicherter Befunde ist in Bezug auf die Lage nur zu sagen, $\mathrm{da} B$ sie unten seitlich in der vordersten Nasenhöhle oder im Vorhof zu erwarten wäre.

In unserem Falle war die Zyste ganz außerhalb der Nasenhöhle seitlich vom knöchernen Nasengerüst gelegen.

Neben der Lagebeziehung müssen wir natürlich auch die gewebliche Beschaffenheit der Zystenwand berücksichtigen. Und mit Recht betont Brüggemann den Wert der histologischen Untersuchung der Zystenwand zur Klärung ihrer Herkunft.

Wenn Brüggemann aber aus der histologischen Beschaffenheit der Zystenwand zwischen Zysten, die vom Tränennasenkanal aus und solchen von, bei der Verschmelzung der primären Gesichtsfortsätze liegengebliebenen, ektodermalen Zellverbänden glaubt unterscheiden zu können, so ist zunächst einzuwenden, daß auch das Epithel des Tränennasenkanals ektodermaler Herkunft ist. Es ist daher kaum zu erwarten, $\mathrm{da} \beta$ in beiden Zystenarten wesentlich verschiedene Verhältnisse vorliegen werden. Andererseits könnten bei beiden große Verschiedenheit und Wechsel im Verhalten entsprechend der hochgradigen Differenzierungsfähigkeit des ektodermalen Gewebes nicht so auffallend erscheinen. Jedenfalls kann, da alle diese Bildungen sich vom Zellhaufen embryonaler Form herleiten und ihre Differenzierungsstärke verschieden ist, die histologische Beschaffenheit im einzelnen nicht den Wert besitzen, wie Br üggemann ihn ihr zuschreibt.

Wenn wir zunächst einmal unseren Fall in genetischer Hinsicht unterbringen wollen, so ist also auch die Herkunft vom vorderen Ende des Tränennasenkanals nach Lage und Form der Zyste sehr unwahrscheinlich.

Alles deutet darauf hin, daß es sich hier um eine Gesichtsspaltzyste im Klestadtschen Sinne handelt. Ja noch mehr. Hier wird man geradezu dazu gedrängt, den Zystenursprung noch näher zu umschreiben und anzunehmen, da $\beta$ er von der Vereinigung des primären seitlichen Nasenfortsatzes mit dem Oberkieferfortsatz herzuleiten ist, indem dabei ein Ektodermstreifen in dem Mesodermgewebe liegen geblieben ist, welches die Epithelmauer zwischen den beiden Fortsätzen durchbrochen hat. $\mathrm{Da}$ ja auch die Tränennasenkanalanlage von der 
Furche zwischen den beiden primitiven Bildungen aus entsteht, würde es sich hier also bei dem schon lokalisierten, mehr auswärts gelegenen Ektodermstreifen geradezu um eine Schwesterbildung (Parallelbildung) handeln. Die gewöhnliche normale Anlage des Tränennasenkanals liegt innerhalb, die Mißbildung außerhalb der Deckknochen, also später außerhalb des knöchernen Nasengerüstes. Man wird es weiter verständig finden, daß die Zyste sich vornehmlich nach unten dem oberen Mundvorhof zu entwickelt hat und hier ihre größte Ausdehnung zeigt, weil hier die nachgiebigste Stelle war. Bei einer Zyste vom vorderen Ende des Tränennasenkanals aus müßte man erwarten, da $B$ sie nur in der vordersten Nasenhöhle und am Vorhof sich entwickelt hätte, nicht aber als derartig hoch hinauf reichende und außen liegende Bildung.

In den einschlägigen Lehrbüchern der normalen und pathologischen Anatomie findet man diese Zysten überhaupt noch nicht erwähnt. Dort sind neben den verschiedenen Gesichtsspalten, Fisteln, intrauterin geheilten Spalten, den fissuralen Angiomen und Karzinomen, nur die Dermoide aufgeführt. Besonders interessant sind ja die intrauterin spontan geheilten Gesichtsspalten, von denen Grünberg ${ }^{1}$ ) in seiner Abhandlung über die Nasenmißbildungen eine sehr anschauliche Abbildung mit einem noch deutlich sichtbaren narbigen Streifen in der Haut bringt, die der schrägen Gesichtsspalte entspricht. Die Narbe zieht vom Nasenflügelansatz zum inneren Augenwinkel hin und verdient in genetischer Hinsicht besondere Aufmerksamkeit bei der Beurteilung unseres Falles.

Vergleichen wir nun den histologischen Befund unseres Falles mit dem von Brüggemann in seinem Falle erhobenen, so ergibt sich eine auffallende Übereinstimmung bei beiden. Brüggemann begründet seine Annahme der Zystenherkunft vor allen damit, daß seine Zyste genau die gleiche histologische Beschaffenheit - einschichtiges Zylinderepithel, das durch Einlagerung von Ersatzzellen zwischen die hohen Zylinderepithelien oft zweischichtig erscheint - wie der Tränennasenkanal aufweist. Er verweist dabei auf Rauber-Kopsch: „Der Tränennasenkanal trägt ein hohes zylindrisches Epithel, welches Ersatzzellen zwischen seinen Bahnen Platz läßt und $z$. T. Flimmerhaare trägt. Becherzellen sind ein häufiges Vorkommen." In den meisten Lehrbüchern der Anatomie und Gewebelehre und den einschlägigen Abhandlungen der Augenheilkunde (Merkel, Sobotta, Schaffer, Stöhr, Schirmer u. a.) findet man überall bei der Beschreibung des Trän€nnasenkanals, daß das Epithel zwei- oder auch noch mehrreihig ist und inselartig Flimmerhaare tragen kann. Abgesehen von dem Fehlen des Flimmerepithels in unserem Falle finden wir also hier dasselbe

1) Schwalbe, Mißbildungen. 
gewebliche Verhalten: zweireihiges Flimmerepithel, reichlicher Gehalt an schleimtragenden, becherartigen Zellen mit stark schleimigem Zysteninhalt. Die schon oben in bezug auf die Wandauskleidung ausgesprochene Erwartung hat sich also, soweit unsere Auffassung zutrifft, bestätigt: Bei beiden Zystenarten - mag sie vom Tränennasenkanal oder von Ektodermresten, die bei der Vereinigung der Gesichtsfortsätze liegen geblieben sind, entstehen - sind diese ben Verhältnisse anzunehmen. Ja nach unserer Auffassung handelt es sich geradezu um eine teilweise Doppelbildung des Tränennasenkanals und mithin dürfte eine differentialdiagnostische Klärung auf dem Wege der histologischen Beurteilung unwahrscheinlich sein. Weder der Gehalt an Becherzellen noch an Flimmerzellen kann wohl entscheidend bewertet werden.

Betrachten wir diese Verhältnisse vom allgemein pathologischen Gesichtspunkte aus, so müssen wir sowohl die Tränennasengangszysten wie die fissuralen Gesichtsspaltzysten als einfache teratoide Zysten, als Hamartome, auffassen. Diese können aber in ihrer Auskleidung bald dem Bau der äußeren Haut (haarhaltigc Epidermisauskleidung, Dermoide), bald den der Schleimhaut aufweisen. So sieht man in unserer Zyste ja neben wechselnd hohem Zylinderepithel auch Inseln mit Plattenepithelcharakter.

Wann bei diesen Hamartomen der Aufbau der äußeren Haut und wann der der Schleimhaut $\mathrm{zu}$ erwarten ist, und warum bei diesen Gesichtsspaltzysten bislang fast ausschließlich die letztere Form beobachtet worden ist, entzieht sich unserer Kenntnis. Ebenfalls steht es noch dahin, warum demgegenüber sich die Zysten in der Orbita, wo sie als Dermoide aufzutreten pflegen, - anders verhalten, als diejenigen am Naseneingang. Weitere sorgfältige histologische Untersuchung der Zysten lassen auch vielleicht bei dieser Form bisweilen Multipotenz der Differenzierung erkennen.

Hier könnte man auch versucht sein, auf die branchiogenen Zysten (tonsillare Kiemengangszysten) zu verweisen, wo es sich auch ganz vorwiegend um Schleimzysten mit einfachem Bau handelt, die mit Zylinder- und Flimmerepithel ausgekleidet sind und serös schleimigen Inhalt haben, seltener um dermoidale Bildungen. Allerdings liegen hier die entwicklungsdynamischen Vorgänge anders als bei den Gesichtsspaltenzysten. Auch ist neuerdings von Weglowski ihre Abstammung vom Ductus thymopharyngeus herzuleiten versucht. Aber immerhin ist ein Vergleich mit Hinblick auf die Differenzierungsneigung interessant. Auch an ihnen ist, abgesehen von gelegentlichen Knorpeloder Knochengehalt $\mathbf{u}$. a. in ihrem Balge, keine besondere Differenzierungsneigung beobachtet, wie sie ektodermale Hamartome oder Choristome nicht selten aufweisen. 
Zur Beurteilung der Zystenherkunft wird also eine sorgfältige Feststellung der Lageverhältnisse und Beziehungen, besonders auch bei der operativen Entfernung, und die histologische Untersuchung der Zystenwand in Blockserien zu fordern sein. Bei großen entwicklungsgeschichtlich zu erklärenden Zysten kann die nähere Bestimmung unmöglich sein, da die Lagebeziehung, die die wichtigsten Anhaltspunkte bietet, dann unsicher wird. Erst auf Grund einer größeren Kasuistik unter Beachtung der hervorgehobenen Kriterien sorgfältig beobachteter Fälle, werden wir mehr Klarheit und Sicherheit in genetischer Beziehung erwarten dürfen. 